\title{
THE EFFECT OF RESERPINE IN COARCTATION OF AORTA
}

\author{
BY \\ A. ACTIS-DATO AND E. DE GIORGI \\ From the Department of Cardiovascular Surgery of the University of Torino, Corso Polonia 14, Torino, \\ Italy
}

Received February 1, 1962

Much information has accumulated in recent years upon the activity, mode of action, and clinical application of Rauwolfia alkaloids in hypertension. However, many problems still remain to be solved. In particular, little is known concerning the effects of this drug on patients with coarctation of the aorta.

The present study has been pursued with the following aims: (i) to evaluate the subjective and objective effects of reserpine upon this condition; (ii) to analyse the possible correlation between the results of the treatment and the different anatomical and hæmodynamic patterns; and (iii) to discover whether some information on the mechanism of action of the drug could be inferred from the response to treatment of patients showing various hæmodynamic derangements.

\section{SUBJECTS AND Methods}

Fifteen patients, aged 17 to 49, were investigated (Fig. 1). The diagnosis was made by clinical, x-ray, and aortographic examinations. Special emphasis was placed on aortography, because by this method associated anomalies are easily discovered while the existence of the stenosis and the presence and distribution of collateral circulation can be closely defined.

Operation was performed in almost all patients and the anatomical data, obtained during the procedure, have been compared with the hæmodynamic pattern recorded previously. Once the diagnostic procedures had been carried out the patients were maintained under conditions of rest though not confined to bed. There was no dietetic restriction and no sedatives or other drugs were given. Blood pressure readings were taken by the classic manometer cuff method while oscillometric and oscillographic recordings were made in the arms and legs. Right arm pressures were preferred to avoid possible errors due to anomalies of implantation of the left subclavian artery. Once the basal conditions were defined, reserpine was started.

Reserpine was administered, $0.5-0.75 \mathrm{mg}$. daily, divided in two or three doses of $0.25 \mathrm{mg}$. The administration was prolonged for some weeks. Blood pressure readings, at the arms and legs, were taken twice daily. Periodically rheographic, oscillographic, and plethysmographic recordings were made. In some cases, synchronous recordings of humeral and femoral pressure were made by direct arterial puncture.

\section{RESULTS}

The best effects were obtained with $0.5 \mathrm{mg}$. reserpine daily, given in two doses of $0.25 \mathrm{mg}$. In all patients, a feeling of calmness was experienced. Headache, complained of by 12 patients out of 15, disappeared or its intensity and frequency appeared less after two to five days of treatment. Coldness of the legs, present in four patients, disappeared on the second to third day of treatment. Præcordial pain, present in 10 patients, disappeared after two to five days of treatment.

Epistaxis was not observed in any patient during reserpine treatments although some of them had been subject to frequent nose-bleeds before admission. Troublesome side-effects were not observed: slight nasal congestion was complained of in four cases, and a feeling of tiredness in three. The reduction in systolic and diastolic blood pressure following reserpine treatment began on the first or second day of treatment. 

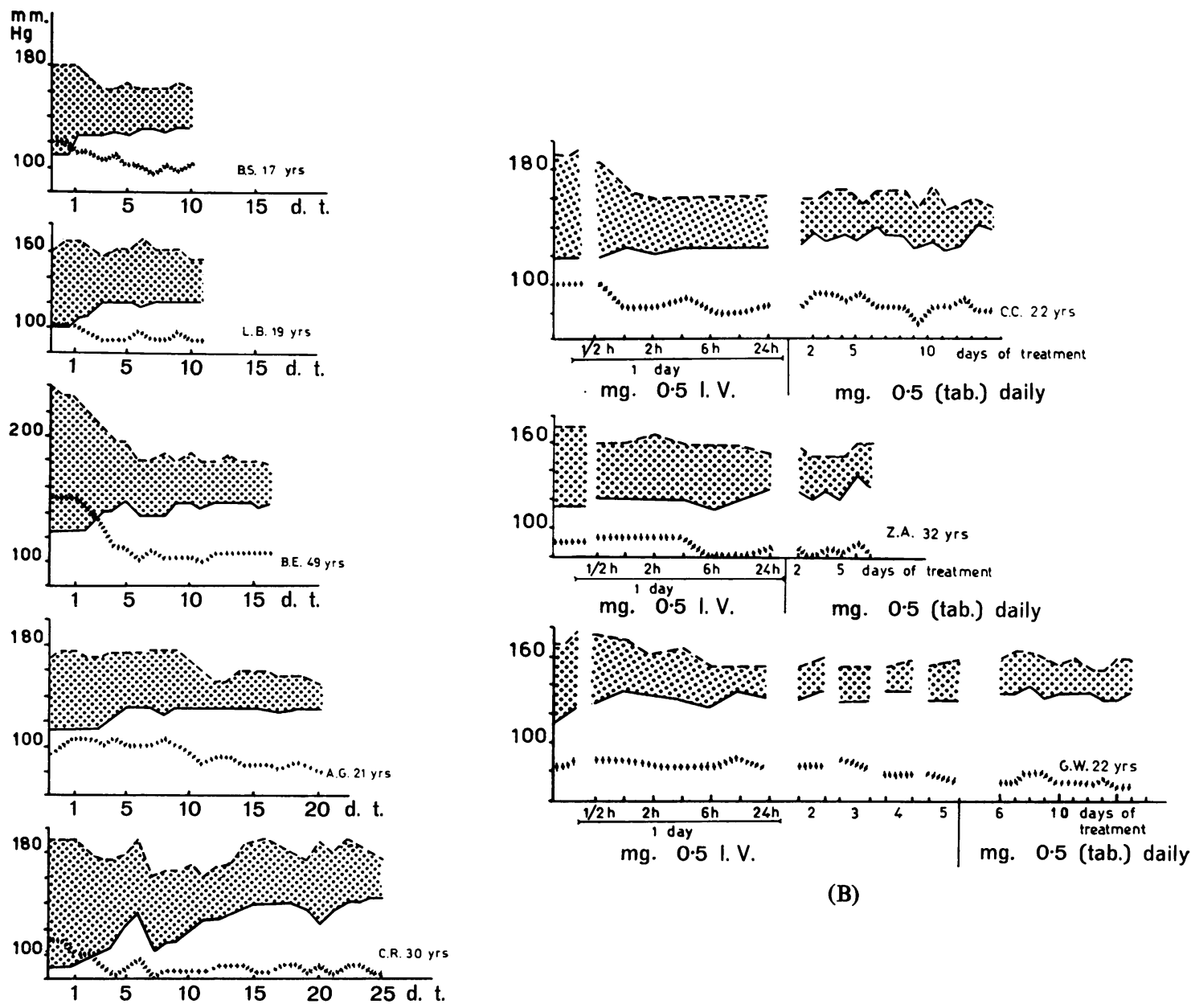

(B)

(A)

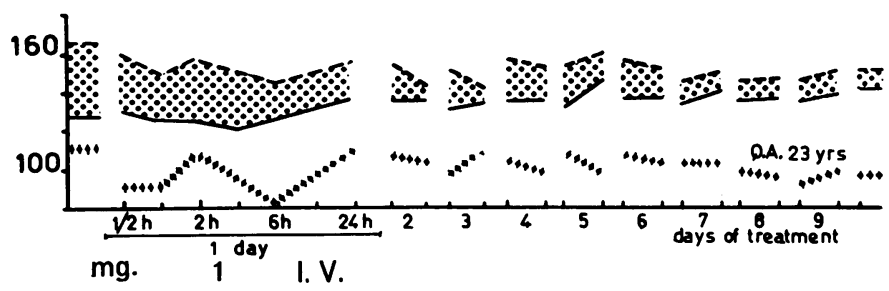

(C)

Fig. 1.-Graphic presentation of pressure changes in nine patients affected by coarctation of the aorta and treated with reserpine. (A) Pressure changes in five patients who had reserpine tablets only. (B) Pressure changes in three patients who had reserpine intravenously at first and later received tablets. (C) Pressure changes in one patient treated by reserpine intravenously only. Shaded areas indicate systolic pressure gradients between arms and legs.

$$
\begin{aligned}
& ---+-- \text { systolic arm blood pressure. } \\
& ++++++ \text { diastolic arm blood pressure. }
\end{aligned}
$$


The fall of both systolic and diastolic brachial pressure was greater if the drug was administered intravenously, this difference being observed in four cases. The greatest fall of both systolic and diastolic blood pressure occurred in two cases within the fifth and eighth day of treatment, in three cases on the fifteenth day, in four cases within the twentieth and twenty-fifth day, in five cases on the tenth day, in one case within the thirtieth day. In general the patients with the highest basal levels had the greatest reduction.

A rise in the systolic blood pressure in the legs began after two or three days of reserpine therapy: in two cases it occurred by the fourth day, in two on the fifth day, in two on the twenty-fifth day, in three on the fifteenth day, and in six cases within the sixth and eighth day of treatment.

The rise in the systolic blood pressure recorded at the femoral artery was more pronounced, generally, in the patients with lowest pressures before treatment. The systolic arm-leg pressure gradient began to decrease on the second or third day of treatment, to reach a minimal value on the sixth-seventh day in three cases, on the tenth-fifteenth day in seven cases, on the eighteenth day in two, and on the twenty-fifth day in two cases. The decrease of the systolic pressure gradient was greatest in those cases in which it was highest before treatment.

On the sixteenth and eighth day of reserpine treatment, two patients with an initial femoral pressure of 80 and $110 \mathrm{~mm}$. $\mathrm{Hg}$ and a systolic pressure gradient of 120 and $70 \mathrm{~mm}$. $\mathrm{Hg}$ respectively, felt for five and seven days intense pain in the legs, which they likened to diffuse needle punctures, simultaneously with the maximal reduction of systolic pressure difference between arms and legs. These symptoms, which subsequently completely disappeared, were considered to be due to the increase of blood flow through a field at first poorly vascularized.

\section{DisCUSSION}

Of particular interest are the correlations between the anatomical situation and the effects of the reserpine treatment. The greatest reduction of the arm-leg pressure gradient (with decrease of brachial pressure and increase in femoral pressure) was observed in those patients who had an anatomically well-developed collateral circulation. On the contrary, poor results from medicinal treatment were obtained in those patients who at operation did not show a good collateral circulation. It was noteworthy that in most patients who benefited from reserpine treatment and showed good collateral circulation at operation, opacification of the post-stenotic part of the aorta was not visible before treatment: in these cases evidently a good collateral circulation was already present before operation, but it was not functionally significant; after reserpine treatment a large dilatation of the collateral vessels took place and contributed to an effective bypass of the aortic obstruction.

This observation further confirms the inference that the collateral circulation is the site of action of Rauwolfia alkaloids in coarctation of the aorta. The foregoing observations have led us to conclusions on the clinical significance and the practical advantages of Rauwolfia treatment. This medication is extremely useful in coarctation of the aorta, not only in those patients who cannot undergo surgical treatment, but also as a pre-operative measure. The patients who had received this treatment showed a more rapid decrease of arm-leg pressure gradient after operation and a better post-operative course.

Reserpine treatment is also useful in those patients, particularly children less than 4 or 5 years old, in whom operation must be delayed for some time. Children in the first years of life affected by coarctation of the aorta complicated by other malformations or aggravated by total cardiac failure can also benefit from reserpine administration, in preparation for surgical correction.

Reserpine therapy can benefit patients afflicted with coarctation, who are in middle age and in whom surgical treatment is not feasible because of the presence of cardiac failure or of associated valvular disease, particularly aortic valvular disease, or of the atherosclerotic lesions particularly frequent in this disease.

The improvement of circulatory conditions and the reduction of cardiac work should lessen the incidence of cerebrovascular accidents due to hypertension or ruptured aneurysms and of cardiac 
failure, complications frequently responsible for death in these patients. It should also increase the expectation of life whether or not surgical treatment is undertaken.

\section{SUMMARY}

Fifteen patients with coarctation of the aorta were treated with reserpine, and the arterial pressure changes in the arms and legs were studied.

In all of them a decrease of systolic blood pressure in the arms was noted and, concomitantly, a progressive increase of systolic blood pressure in the legs.

It is probable that the drug induces a dilatation of the system of vessels bypassing the point of narrowing, especially in those patients having a well-developed collateral circulation.

The data are compared with the aortographic data and the surgical findings. The clinical data have been correlated with those obtained by aortography and at operation.

Our thanks are due to Messrs. Ciba Industria Chimica, Milano, for a generous supply of Serpasil (reserpine). 